\title{
Lateral Ridge Augmentation by Titanium Membrane Using Autograft and Alloplast: A Case Report with 3 years Radiological Follow-up
}

\author{
${ }^{1}$ Lanka Mahesh, ${ }^{2}$ Manesh Lahori, ${ }^{3}$ Praful Bali, ${ }^{4}$ Sunanda Choudary \\ ${ }^{1}$ Private Practice, New Delhi, India \\ ${ }^{2}$ Professor, Department of Prosthodontics, KD Dental College, Mathura, Uttar Pradesh, India \\ ${ }^{3}$ Private Practice, Bali Nursing Home, DB Gupta Road, New Delhi, India \\ ${ }^{4}$ Professor and Head, Department of Orthodontics, Sri Bankey Bihari Dental College, Uttar Pradesh, India \\ Correspondence: Lanka Mahesh, The Dental Centre, S-382, Panshila Park, New Delhi-17, India \\ e-mail: drlanka.mahesh@gmail.com
}

\begin{abstract}
Purpose: The objective of the present study was to clinically evaluate the effectiveness of autograft and alloplast combined with a titanium membrane for lateral ridge augmentation.

Material and methods: Autograft and allograft as an augmentation material, in conjunction with titanium membrane.

Results: Bone defects of $3.5 \mathrm{~mm}$ can be augmented by using autografts and allografts. Bone width gained at 5 months after augmentation was $6 \mathrm{~mm}$.
\end{abstract}

Keywords: Autogenous bone grafts, alloplasts, titanium membrane, lateral ridge augmentation.

The maxilla is the most challenging region of the dentition to restore. In many cases due to trauma, loss of bone volume from periodontal disease, neoplasms, or long-term edentulism, alveolar ridge augmentation is necessary for proper positioning of implants. ${ }^{7}$ Bone grafting to support esthetics is key in this prominent area as the underlying ridge must support both the implant restoration and the soft tissue to achieve a long-lasting, esthetic result. Lateral ${ }^{1}$ ridge augmentation is the challenge in bone construction. Relevant lateral defects of alveolar ridge may render the use of dental implants unfavorable. Severe alveolar bone resorption can occur after tooth removal. ${ }^{8}$ Treatment using the placement of dental implants requires bone height and width. When these conditions are not present, additional surgical treatment is necessary to create adequate bone volume. Different surgical technique can be performed to reconstruct the alveolar ridge. These techniques range from guided bone regeneration, autogenous and allograft bone block, to distraction osteogenesis. Mandibular fracture has been reported during ramus and chin grafting. ${ }^{13}$

Guided bone regeneration is associated with the use of a particulate bone graft material and membranes. Autogenous block grafts can be harvested from intraoral sites, e.g. chin $^{2-5}$ or ramus or extraoral sites, e.g. iliac crest. Each of these techniques has their own advantages and disadvantages. Harvest of autogenous bone from these sites require a second surgery site and involves significant risk of neurological and vascular adverse events and postsurgery sequale. ${ }^{9-12}$

Other unique problems that may limit ideal placement of implants in the maxilla include:

1. A resorbed (from periodontal disease) or fractured (from extraction) facial cortical plate over the maxillary roots of teeth.

2. Close proximity of the nasal and maxillary sinus cavities.

3. Lateral extension of the incisive canal.

4. Facial concavities.

\section{CASE REPORT}

A 24-year-old male patient reported to the dental office desiring replacement of upper left posterior teeth, he was in good overall health with no systemic disorders.

Initial intraoral examination revealed a large buccal defect on visual (Fig. 1) as well as bone gauge examination, (Fig. 2), all treatment options including autogenous bone grafting and endosseous. 


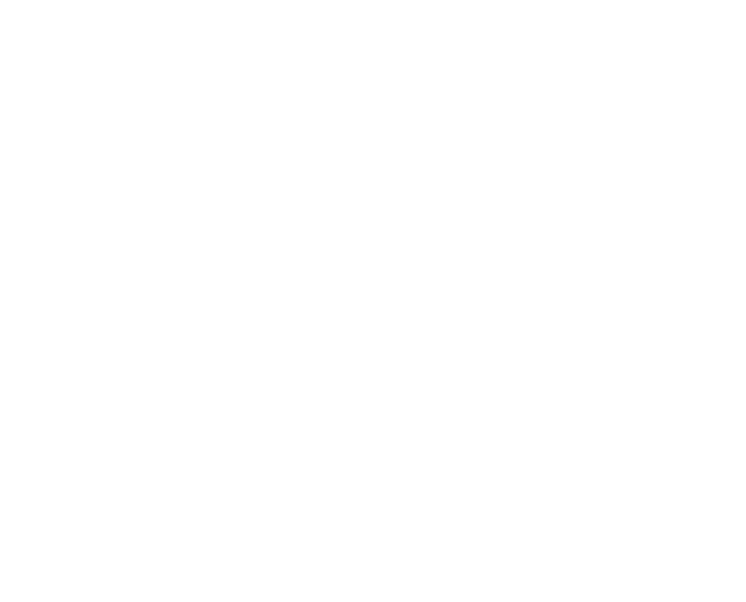

Fig. 1: Defect prior to intervention

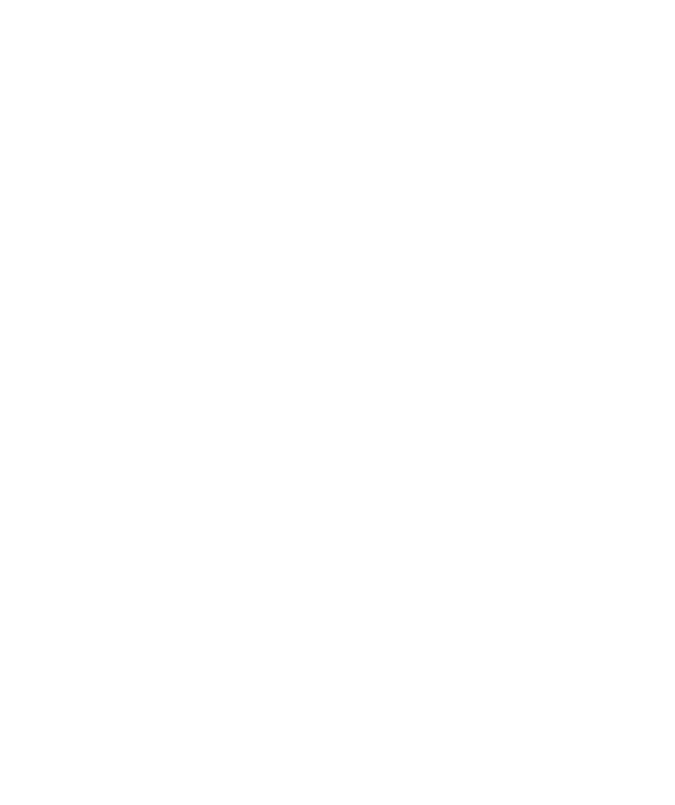

Fig. 2: Use of bone gauge for measuring the bone defect

Implants were discussed in detail and the patient opted for the same. Medical history of the patient did not reveal any significant systemic conditions which would interfere with the operative procedure.

\section{SURGICAL PROTOCOL}

The patient received antibiotic prophylaxis (amoxicillin and clavulanic acid, $1 \mathrm{gm}$ every 8 hours, starting 1 day preoperatively) in association with $0.2 \%$ chlorhexidine gluconate mouth rinse every 12 hours, starting 1 day preoperatively for 7 days).

Local anesthesia was combined with a sedative premedication (diazepam, $5 \mathrm{mg}$ administered orally 30 minutes before surgery). A full thickness incision was made within the keratinized gingiva from the distal aspect of \#23. The incision was extended intrasulcularly and anteriorly to \#27. Vertical releasing incisions were made at the mesiobuccal line angle of the second to last existing tooth and at the distal aspect of the crestal incision. The buccal and lingual flaps were reflected with a back action chisel, JO $1(\mathrm{Hu}$ Friedy, $\mathrm{CH}$, USA), avoiding damage to the anatomic structures. Tooth number 23 and 26 were extracted and once exposed, the cortical bone was curetted with a backaction chisel to remove all residual connective tissue and the periosteum. Bone was obtained from chin area using Trephine burs (Meisenger, Germany Fig. 3) and was mixed with , Frioss Algipore (Friadent Gmbh) in a bone mill.

Decortications of the ridge was performed to activate the regional accelatory phenomenon.

The bone was placed laterally over the defect and was stabilized by placing titanium membrane Osseoshield (Friadent, Gmbh) by means of membrane tacks, Auto Tac (Biohorizons, AL, USA). Flaps were sutured with a combination of horizontal mattress and simple interrupted sutures using 3-0 cytoplast suture (Osteogenics Biomedical, TX, USA).

The patient was instructed to use chlorhexidine mouthwash $0.12 \%$ twice a day for a month. He was also instructed to avoid brushing and trauma to the surgical site. Analgesic Diclomol (Win Medicare India) three times a day for 5 days was prescribed to the patient. Sutures were removed after 2 weeks, and then postsurgery appointments were at 4, 6, 8 and 12 weeks for evaluation of healing.

The site was re-entered for implant placement 5 months after the ridge augmentation. A midcrestal incision was made

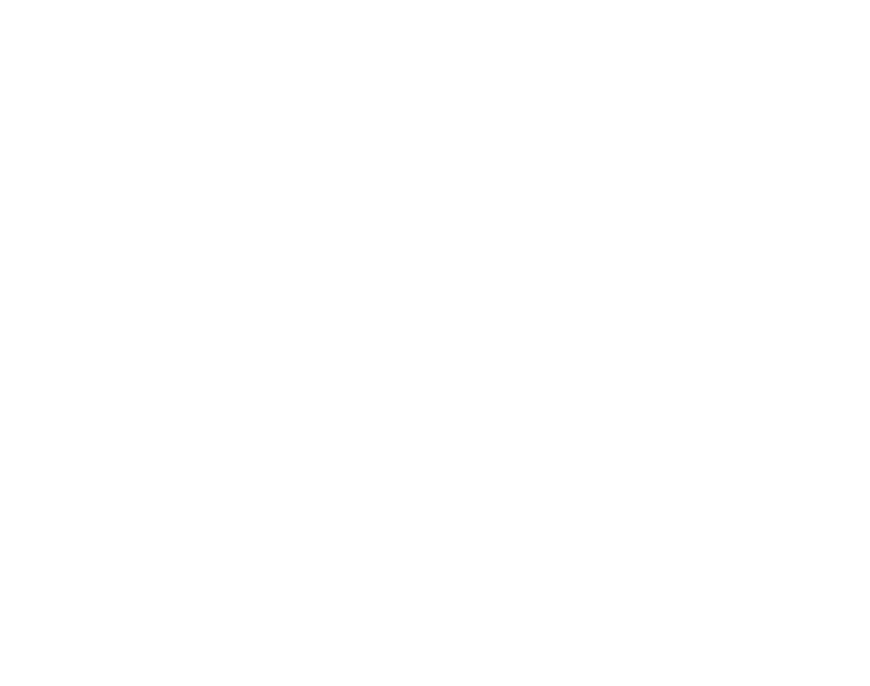

Fig. 3: Trephine harvest of chin graft 


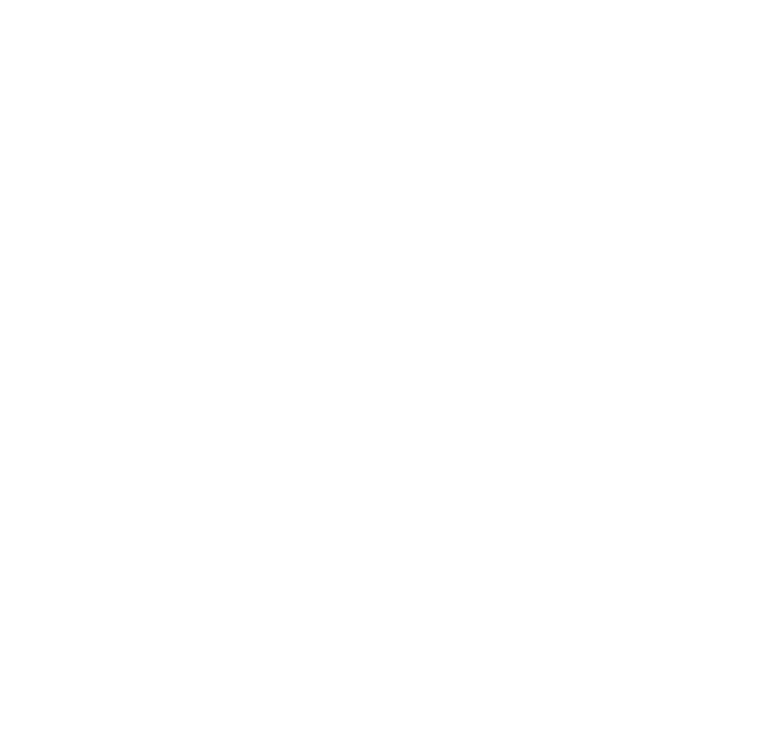

Fig. 4: Re-entry surgery for placement at 5 months

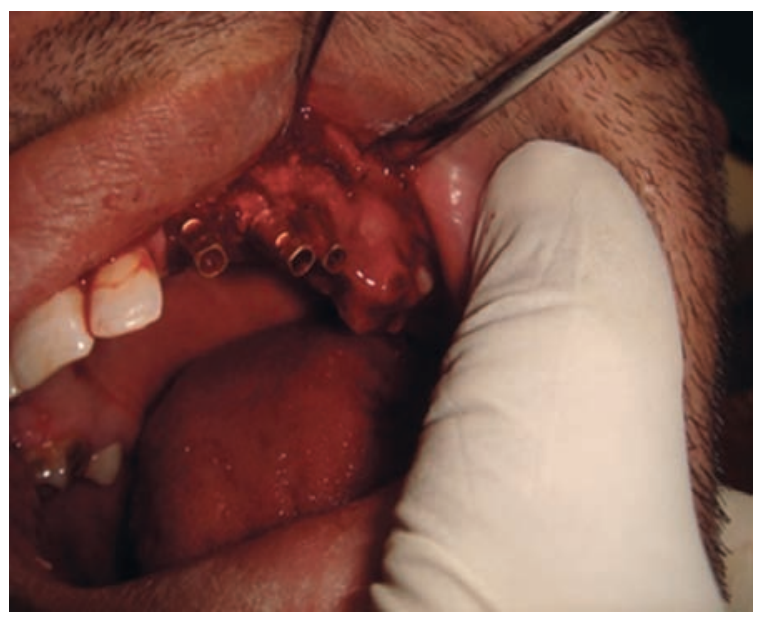

Fig. 5: Implant placement

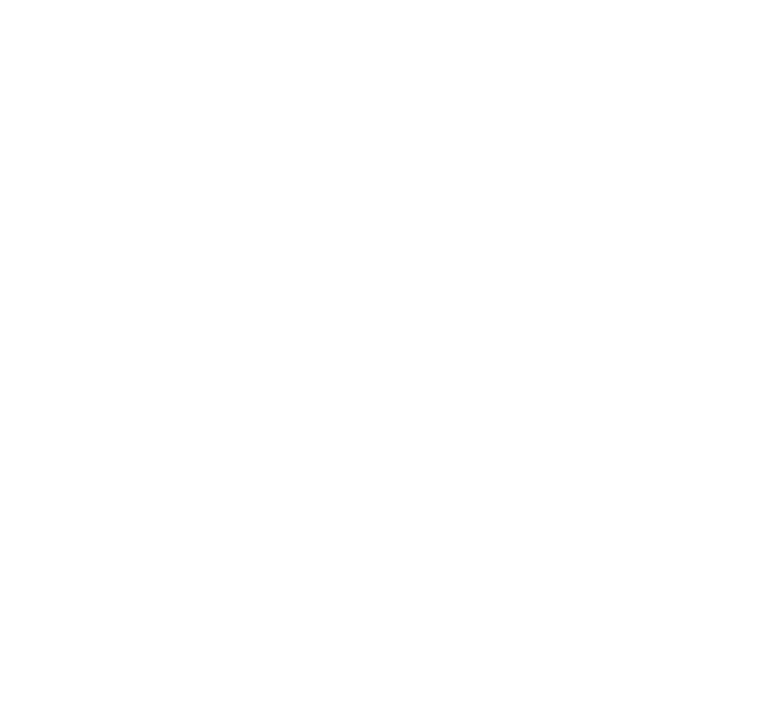

Fig. 6: X-ray at implant placement and 2 vertical releasing incision were given and a fullthickness flaps were elevated to allow measurement of bone augmentation site. The titanium membrane was removed at this stage (Fig. 4).

The autograft and alloplast showed minimal resorption and bone width of $6 \mathrm{~mm}$ was achieved. Using a surgical stent, 3 Biohorizons external implants (AL, USA) of diameters $3.5 \mathrm{~mm}$ and length $12 \mathrm{~mm}$ were placed as per conventional surgical protocol (Fig. 5). The X-ray image at implant placement shows correct equidistant placement of implants (Fig. 6).

The patient returned at 2, 4, 6, and 8 weeks of postsurgery healing.

\section{SECOND STAGE SURGERY}

Due to soft tissue recession and cover screw exposure (Fig. 7), a 4 mm tissue punch (Stoma, Germany) was used at the palate $4 \mathrm{~mm}$ away from the implants to remove tissue at each implant site (Fig. 8). A partial thickness flap was elevated and advanced buccally (Figs 9 and 10). The healing collars were placed (Fig. 11) and the site was sutured with a combination of 3-0 Cytoplast (Osteogenics Biomedical, TX, USA) and 3-0 chromic Gut (Ethicon, Johnson and Johnson, India) (Fig. 12).

After 3 weeks impressions were recorded in a closed tray technique using PVS material (Honigum, DMG), a cement retained PFM prosthesis was delivered (Fig. 13).

The final radiograph at 3 years postoperative shows minimal crestal bone loss (Fig. 14).

\section{RESULTS}

During the 5 months healing period after placement of the autograft and allograft, no membrane exposure or infection of the site was seen. On re-entry for implant placement, the Osseoshield was found to be intact and it completely covered the graft.

At re-entry for PME placement, the implants were integrated as they produced typical "ring"7 when tapped with a mirror handle. The implants were restored with a cement retained PFM bridge. As of this report, implants placed into this augmented site have been in function over 36 months, with no complications.

\section{DISCUSSION}

The present clinical case help us to evaluate whether this technique for horizontal bone regeneration using 


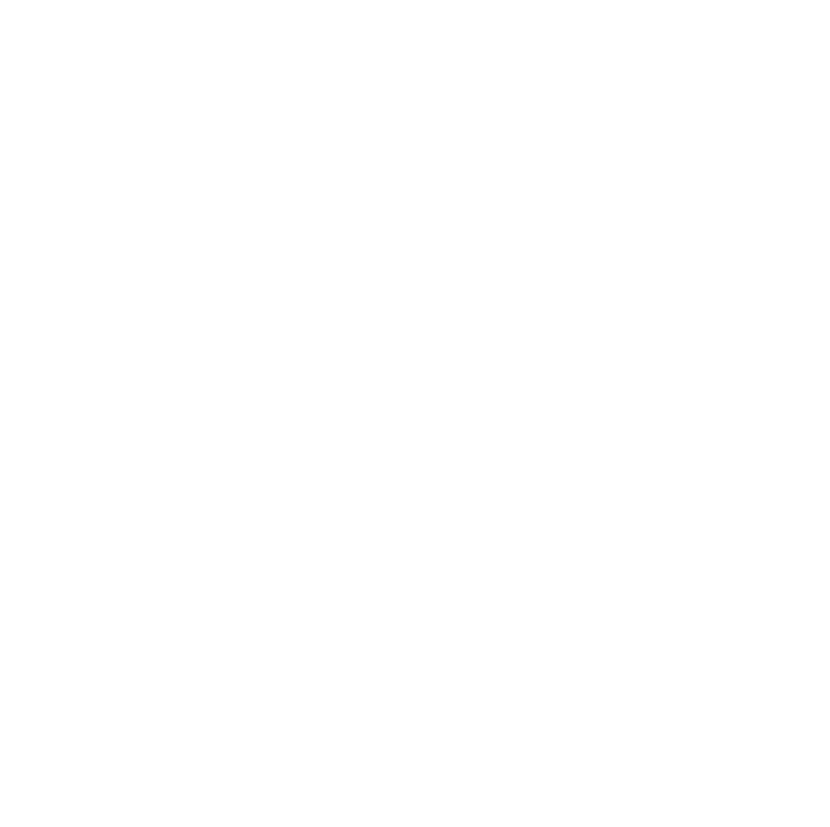

Fig. 7: Five months postoperative healing

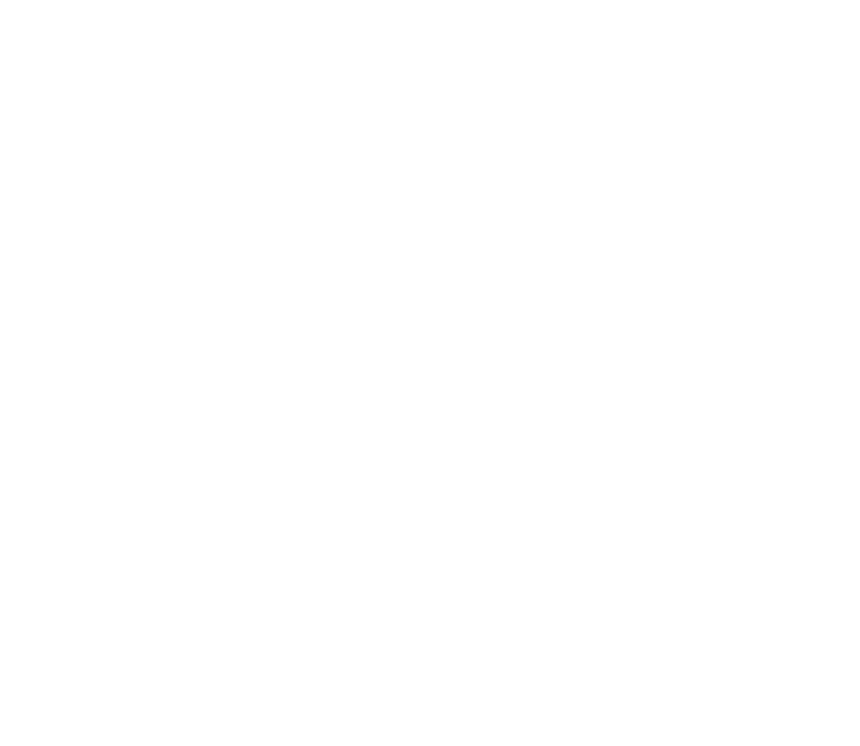

Fig. 8: Use of tissue punch palatally

combinations of particulated autogenous bone graft (chin graft) and alloplast (Algipore-Friadent, Gmbh) graft which are covered by titanium reinforced membrane (OsseoshieldFriadent, Gmbh) could offer some advantage in lateral ridge augmentation.

Labial ridge deformity can be more severe if there is damage to the buccal plate during tooth removal. Bone

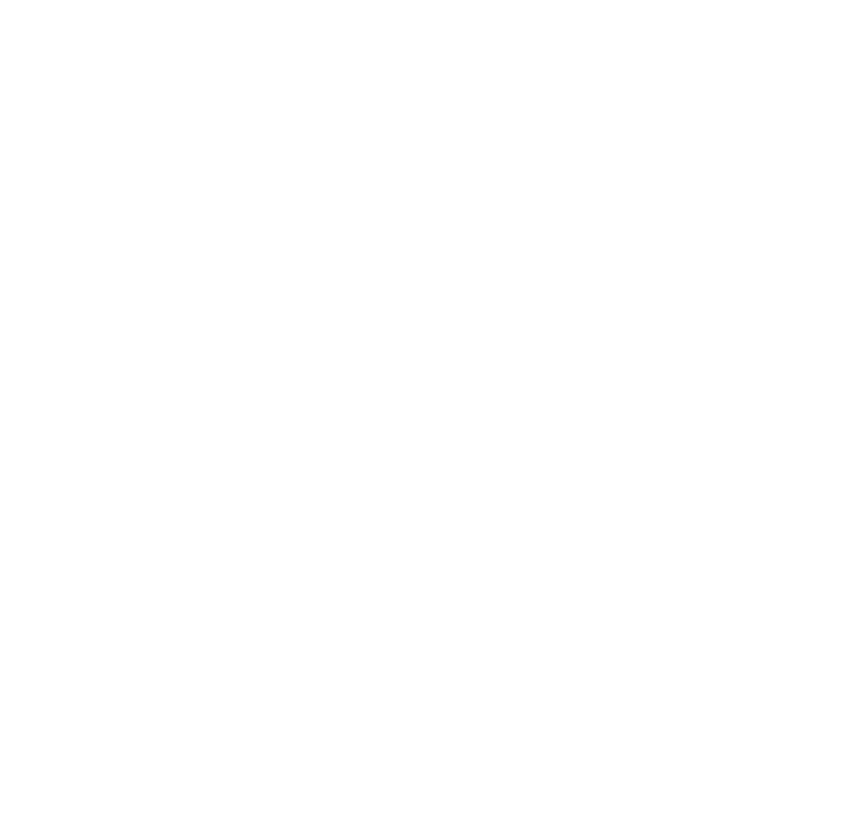

Fig. 9: Elevation of palatal flap

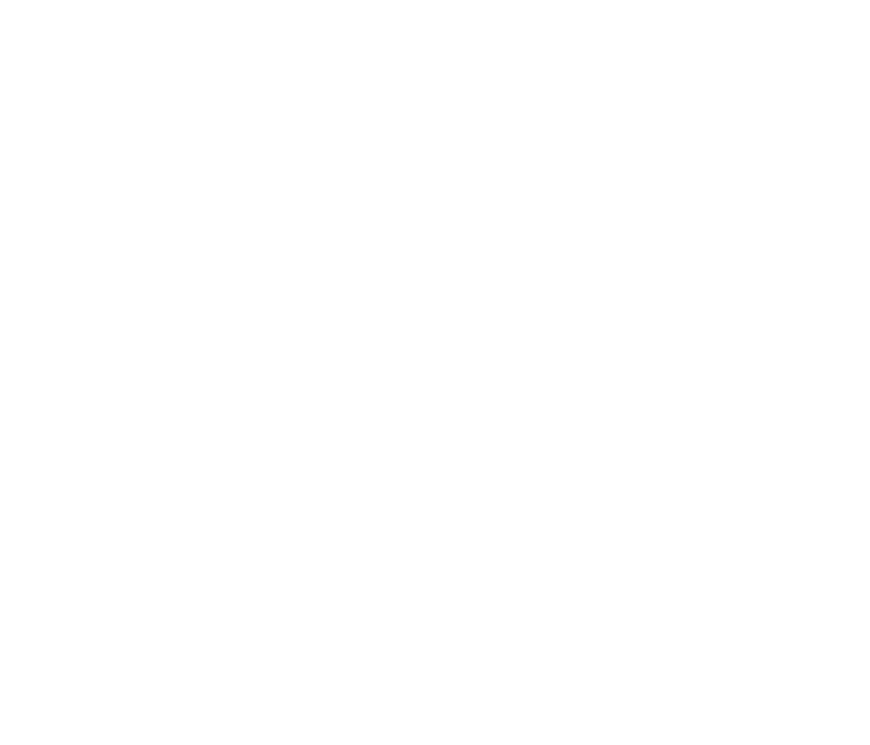

Fig. 10: Completion of flap elevation

resorption activity continues throughout the life, as a result it is anticipated that many patients with tooth loss will have varying degrees of alveolar ridge resorption. This resorption can lead to compromise in the position of the implant fixture. In addition, the importance of having adequate labial bone thickness around implants cannot be underestimated. Particulate bone graft may be better alternative, because it 


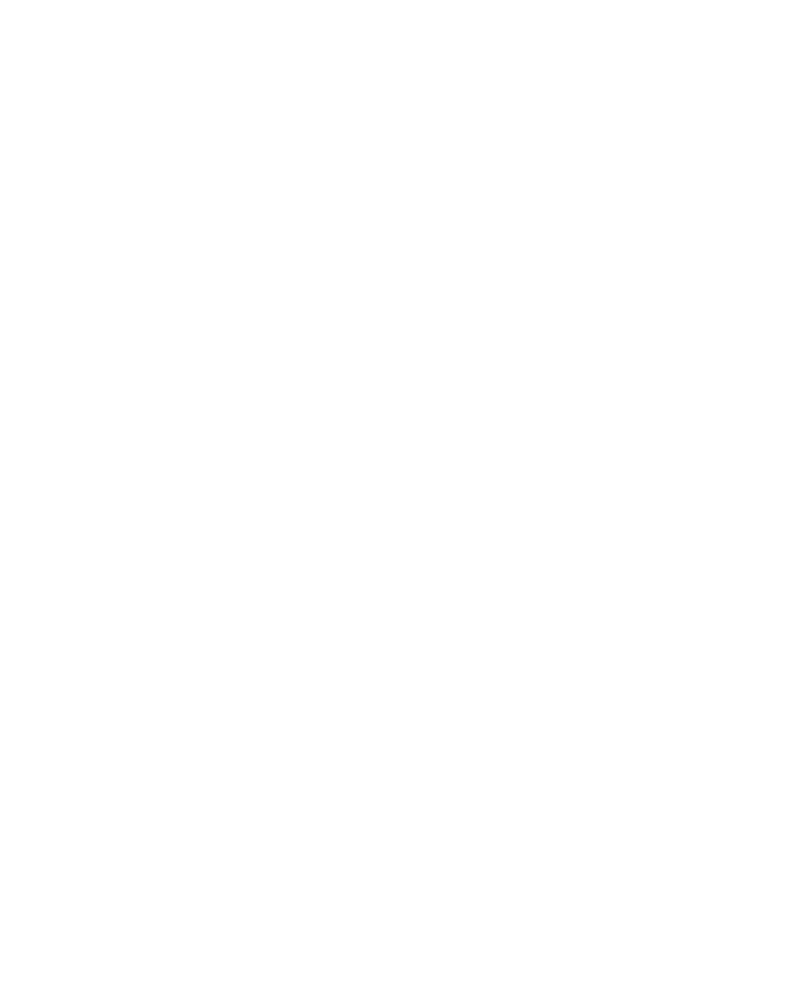

Fig. 11: Flap rolled buccally and healing collars/pme's placed

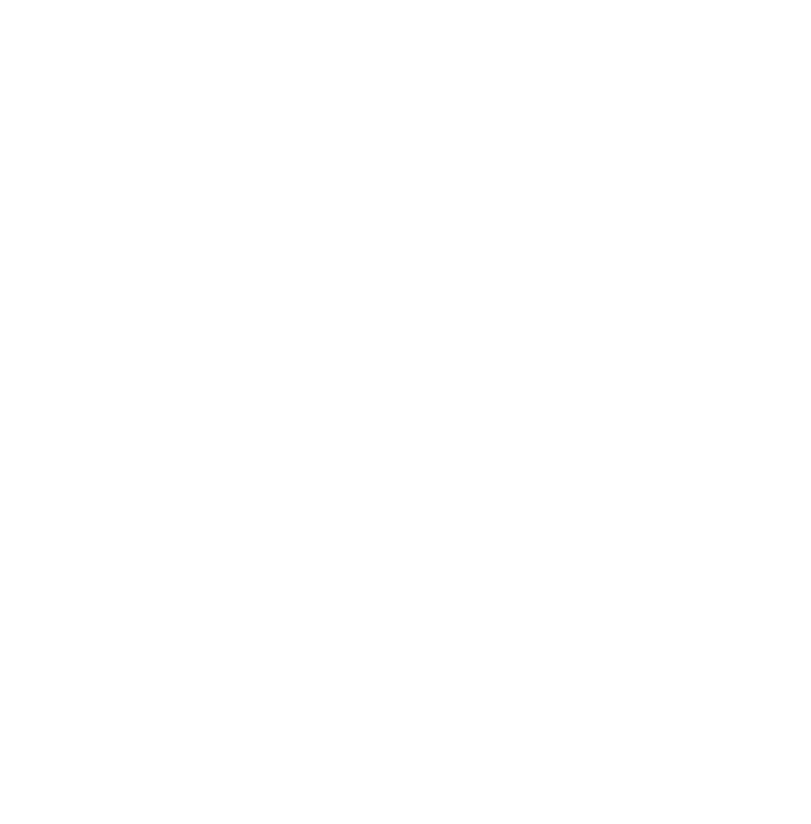

Fig. 12: Sutured flap

treats the underlying bone defect to restore the natural support of the tissue architecture. The result in this case report suggest that particulated autograft can be combined with allograft under a regenerative membrane for significant

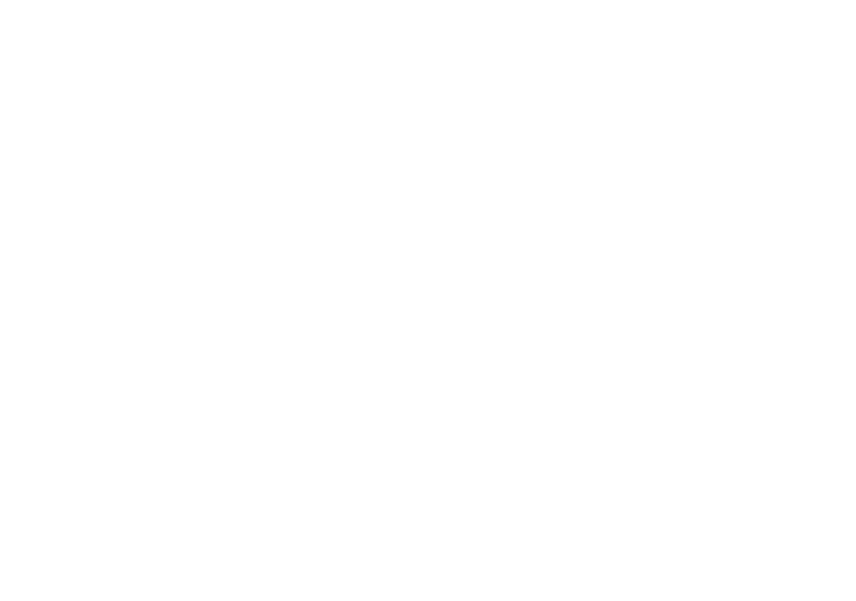

Fig. 13: Final clinical view

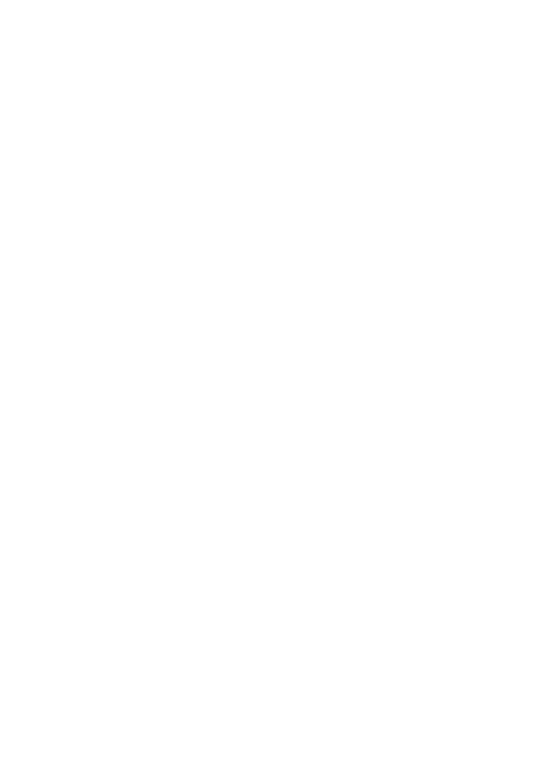

Fig. 14: Radiograph at 3 years

lateral ridge augmentation. Studies have reported bone resorption approximately 7 times greater without a membrane than with a barrier membrane over autograft and allograft. ${ }^{14}$ The presence of intact osseoshield (titanium membrane) suggests that this type of membrane can stay intact for a much longer period of time. This longevity aids in prevention of resorption of allograft and maximize the resulting volume of newly formed bone. There are significant differences in the healing process of autogenous cortical versus autogenous cancellous grafts. ${ }^{15}$ Cancellous bone revascularizes faster and is strengthened first by creeping substitution; whereas cortical grafts revascularizes slower and remain a mixture of necrotic and living bone for prolonged period of time. There are also important differences in mechanical strength from the repair process. 
Cancellous grafts are strengthened during the repair process, whereas cortical grafts are weakened during repair. ${ }^{16}$

\section{CONCLUSION}

This clinical report suggests that lateral ridge augmentation with autograft, allograft and titanium membrane is predictable and can lead to long-term success. Particulate onlay grafts can be used to convert unhealthy and unesthetic gingival contours into favorable sites. Cancellous grafts are strengthened during the repair process, whereas cortical grafts are weakened during repair. ${ }^{6}$ In reviewing the various techniques used to develop the implant site at the buccal aspect of the ridge, this technique made implant insertion possible and improved implant esthetics too. Correction of labial defects is just one of the many factors leading to excellent esthetic results. Just as important are treatment planning and case selection, correct implant placement, proper abutment selection and esthetic fabrication of the final crown. A longer follow-up is needed to evaluate the long-term stability of this grafting technique described.

\section{REFERENCES}

1. Buser D, Dula K, Hirt HP, et al. Lateral ridge augmentation using autografts and barrier membranes. J Oral Maxillfac Surg 1996.

2. Pikos MA. Facilitating implant placement with chin grafts as donor sites for maxillary bone augmentation-Part I. Dent Implantol Update 1995;6:89-92.

3. Hunt D, Jovavanovic S. Autogenous bone harvesting: A chin technique for particulate and monocortical bone blocks. Int J Periodontics Restorative Dent 1999;19:165-73.

4. D'Addona A, Nowazari H. Intramembranoous autogenous osseous transplants in aesthetic treatment of alveolar atrophy. Periodontaol 2000;2001;27:148-61.
5. Garg AK, Morales MJ, Navarro I, et al. Autogenous mandibular bone grafts in the treatment of the resorbed maxillary anterior alveolar ridge: Rationale and approach. Implant Dent 1998;7: 169-76.

6. Goldberg VM, Stevensons S. Natural history of autografts and allografts. Clin Orthop 1987;225:7-16.

7. Stephen W, Robert Gellin. Clinical evaluation of a cancellous block allograft for ridge augmentation and implant placement: A case report. Implant Dentistry 2008;151-54.

8. Johnson K. A study of the dimensional changes occurring in the maxilla following tooth extraction. Aust Dent J 1969;14:241-44.

9. Bahat O, Fontanesi FV. Complications of grafting in the atrophic edentulous or partially edentulous jaw. Int J Periodontics Restorative Dent 2001;21:487-95.

10. Nkenke E, Schultze-Mosgau S, Kloss F, et al. Morbidity of harvesting of chin grafts: A prospective study. Clin Oral Implants Res 2001;12:495-502.

11. Raghoebar GM, Louwerse C, Kalk WWI, et al. Morbidity of chin harvesting. Clin Oral Implants Res 2001;12:503-07.

12. Ahlmann E, Patzakis M, Roiddis N, et al. Comparison of anterior and posterior iliac crest bone grafts in terms of harvest-site morbidity and functional outcomes. J Bone Joint Surg Am 2002;84:716-20.

13. Pikos M. Academy of oseeointegration annual meeting, San Antonio: Texas, March 2007;11.

14. Antoun H, Sitbon JM, Martinez H, et al. A prospective randomized study comparing two techniques of bone augmentation: Onlay graft alone or associated ith a membrane. Clin Oral Implants Res 201;12:632-39.

15. Burchardt H. The biology of bone graft repair. Clin Orthop 1983;174:28-42.

16. Goldberg VM, Stevenson S. Natural history of autografts and allografts. Clin Orthop 1987;225:7-16. 